\title{
Geolocalizando con TAC: La Competencia Digital Docente y la Competencia Espacial con TPACK
}

\section{Geolocalizing with LKT: Digital Teaching Competence and Space Competence with TPACK}

Isabel María Gómez-Trigueros

isabel.gomez@ua.es (1) https://orcid.org/0000-0003-4666-5035

Facultad de Educación, Universidad de Alicante. Calle Aeroplano, s/n. 03690 San Vicente del Raspeig, Alicante. España.

\section{INFO ARTÍCULO}

Recibido: 08-04-2020

Revisado: 05-05-2020

Aceptado: 22-05-2020

\section{PALABRAS CLAVE}

Geolocalización

Competencia Digital Docente

Formación

TAC

Profesorado en formación

\section{KEYWORDS}

\section{Geolocation}

Digital Teacher Competence

Training

TAC

Teachers in training

\section{RESUMEN}

Las investigaciones sobre la capacitación en conocimientos relativos a geolocalización con tecnologías evidencian las potencialidades y las limitaciones formativas que estos recursos ofrecen para profesorado en formación. Este estudio examina las percepciones sobre la efectividad instruccional de los dispositivos móviles y software de geolocalización, mediante el análisis del rendimiento en las dimensiones competenciales TPACK del futuro profesorado $(n=383)$. Se ha implementado una metodología mixta, de enfoque descriptivo transversal así como análisis inferenciales a partir de cuestionarios $(\alpha \geq .903)$ y de las tareas elaboradas por la muestra. Los resultados arrojan un deficiente uso didáctico de las tecnologías al tiempo que constatan la adquisición de conocimientos sobre geolocalización. Se corrobora así las bondades de aplicar una mejora en la competencia digital del profesorado en formación.

\begin{abstract}
Research on training in knowledge related to geolocation with technologies shows the training potentials and limitations that these resources offer for teachers in training. This study examines the perceptions of the instructional effectiveness of mobile devices and geolocation software, by analyzing the performance in the TPACK skills dimensions of future teachers $(n=383)$. A mixed methodology has been implemented, with a descriptive cross-sectional approach as well as inferential analysis based on questionnaires $(a \geq .903)$ and the tasks carried out by the sample. The results show a poor didactic use of technologies while confirming the acquisition of knowledge about geolocation. This corroborates the benefits of applying an improvement in the digital competence of teachers in training.
\end{abstract}




\section{INTRODUCCIÓN}

Las directrices recogidas en la recomendación europea de 2006 (Recomendación 2006/962/CE, 2006) ya ponía de manifiesto la importancia otorgada a las Tecnologías de la Información y la Comunicación (TIC) así como a la adquisición de la competencia digital docente (CDD) en relación con la formación del profesorado. Dicho reconocimiento ha implicado un cambio pedagógico en el que toda Europa y el mundo se encuentran sumidos. Se trata de dar una respuesta contundente a las necesidades relacionadas con la formación del docente en el binomio didáctica-tecnología.

El concepto de competencia recogido en el proyecto de 2003 de la OCDE denominado DeSeCo (Rychen, Hersh \& Userkonstant, 2003) sugiere un conjunto de capacidades, habilidades y/o destrezas básicas, que se constituyen en los aprendizajes imprescindibles para la ciudadanía, enmarcada en la Sociedad de la Información y del Conocimiento (SIC). Entre las competencias clave se encuentran las siguientes: comunicación lingüística (CCL); competencia matemática y competencias básicas en ciencia y tecnología (CMCT); competencia digital (CD); aprender a aprender (CPAA); sociales y cívicas (CSC); sentido de la iniciativa y espíritu emprendedor (SIE) y conciencia y expresiones culturales (CEC) (Mateo, 2010). También se requieren destrezas como la capacidad para la aplicación de los principios y los procesos matemáticos, la resolución de operaciones, las destrezas vinculadas con el tratamiento de la información, la lectura multimodal, la producción de textos y la comprensión de los términos y conceptos espaciales, formales y de datos entre otros. Este último aspecto de la comprensión de conceptos espaciales hace referencia a la denominada habilidad espacial (HE) y al conjunto de capacidades relacionadas con el posicionamiento, la localización y la comprensión del territorio en el área de la geografía.

Aunque no existe un consenso en cuanto a la definición de competencia espacial (CE), los numerosos trabajos que se han ocupado del tema convergen en caracterizarla como la habilidad de representar, generar, recordar y transformar información simbólica no lingüística (Linn \& Petersen, 1985; Vázquez Maris y Noriega Biggio, 2010). La ciudadanía, debe ser capaz de leer, comprender y utilizar un plano o un mapa, para poder orientarse en el espacio. En este sentido, los futuros docentes, que tienen como tarea la formación en competencias de sus estudiantes, no sólo deben contar con la HE para ser transmisores y enseñantes de tales conocimientos, sino que también deben apropiarse de las potencialidades didácticas con la que cuentan las TIC para tal fin. De ahí que, tales circunstancias han provocado el desarrollo de un nuevo paradigma educativo (Gómez Trigueros, 2015; Ortega Sánchez y Gómez-Trigueros, 2017), que precisa de la inclusión de las tecnologías en los modelos educativos y, consecuentemente, la necesidad de desarrollar las competencias y las habilidades del profesorado, en el uso de estos recursos, como instrumentos básicos en su formación (Roig-Vila, Mengual-Andrés, Sterrantino y Quinto, 2015).

La cuestión actual es cómo implementar modelos de enseñanza y aprendizaje (E-A) que permitan aunar contenidos, pedagogías y tecnologías de una manera dinámica e inclusiva. Surgen así diversas opciones que buscan resolver esa compleja simbiosis como el modelo Technological Pedagogical Content Knowledge (TPACK) (Mishra \& Koehler, 2006). Este modelo propone, entre otros aspectos, la conjunción entre el denominado Conocimiento Base del Docente (Shulman, 1987) y los novedosos recursos TIC (Järvelä, Näykki, Laru \& Luokkanen, 2007; Gómez-Trigueros, Ruiz-Bañuls y Ortega-Sánchez, 2019).

En este sentido, este estudio se centra en la consecución de los siguientes objetivos: el primero, evaluar el nivel de competencia geoespacial del profesorado en formación; el segundo, conocer su percepción respecto del uso de las tecnologías para la docencia; el tercero, estudiar y valorar la importancia que otorgan a una capacitación manipulativa, didáctica y pedagógica de los recursos tecnológicos en su formación universitaria y, por último, valorar el progreso en competencia geoespacial al finalizar la intervención.

En la primera parte del artículo se presenta el estado de la cuestión acerca del concepto y evolución de la CE y su imbricación con la disciplina geográfica; la importancia actual de la formación en CDD y los trabajos que se han desarrollado en otras investigaciones. En la segunda parte sintetizamos los principales hallazgos de la intervención implementada, así como las conclusiones alcanzadas. 


\section{ANTECEDENTES TEÓRICOS}

\subsection{La competencia espacial}

Definir el concepto central de esta investigación, "competencia espacial", es fundamental para la comprensión de los resultados que posteriormente se ofrecerán. El diccionario de la Real Academia Española define "capacidad" como aptitud, talento, cualidad que dispone a alguien para el buen ejercicio de algo. La "habilidad" es la capacidad y disposición para algo. El término "competencia" podríamos, grosso modo, asociarlo al concepto de "capacidad" o "habilidad". En este trabajo se ha optado por utilizar el término "competencia espacial" más que "capacidad espacial", siguiendo la línea de los investigadores españoles en este campo.

El marco referencial para definir las competencias lo constituye las declaratorias procedentes de la UNESCO (2007; 2013), la OCDE (2010) y el Informe J. Delors (1996). Por competencia se entiende: "el desarrollo de las capacidades complejas que permiten a los estudiantes pensar y actuar en diversos ámbitos [...]. Consiste en la adquisición de conocimiento a través de la acción, resultado de una cultura de base sólida que puede ponerse en práctica y utilizarse para explicar qué es lo que está sucediendo" (Braslavsky y Acosta, 2006, p. 35). La competencia es pues "una combinación de habilidades prácticas y cognoscitivas interrelacionadas, conocimientos, motivaciones, valores y ética, actitudes, emociones y otros componentes sociales y comportamentales que puedan movilizarse conjuntamente para una acción eficaz en un contexto particular" (Rychen et al., 2003, p. 21).

Los estudios relacionados con la competencia espacial son múltiples y variados. Por competencia espacial (CE) se reconoce un aspecto de la capacidad intelectual (Lohman, 1986; 1989), "que es unitaria en sí misma, pero está compuesta de múltiples subhabilidades que pueden estar más o menos acentuadas en las distintas personas y que influyen en el nivel de logros en diversos campos" (Vázquez Maris y Noriega Biggio, 2010, p. 67). De este modo, las CE se hallan implicadas en la resolución de problemas tales como la interpretación de mapas, entre otras múltiples actividades académicas y de la vida cotidiana. Las primeras concepciones relativas a las inteligencias o capacidades del ser humano se basan en los postulados de autores como Gardner (1983; 1993; 1999; 2006), Larsem-Freeman (2000) o Richards \& Rogers (2001) sobre inteligencias múltiples. Para dichos autores, las inteligencias vendrían a ser "un potencial biopsicológico para procesar información que se puede activar en un marco cultural para resolver problemas o crear productos que tienen valor para una cultura" (Gardner, 1999, p. 45). Por ello, se constata que las inteligencias no son algo tangible, sino que son potenciales (neurales), capacidades que se activan o no en función de diferentes variables (ambiente sociocultural; contexto educativo-formativo; decisiones personales entre otros). Las subinteligencias o "núcleos" están separados entre sí, tienden a entrar en acción conjuntamente y, en consecuencia, su agrupamiento está justificado (Gardner, 2006).

En relación a la inteligencia espacial o CE, esta comprende las capacidades para percibir con exactitud el mundo visual, realizar transformaciones y modificaciones de las percepciones iniciales propias, y recrear aspectos de la experiencia visual propia, incluso en ausencia de estímulos físicos apropiados (Gardner, 1983). Así, esta competencia emerge como amalgama de habilidades, que parten de una operación elemental: la capacidad para percibir una forma u objeto de la realidad (Dziekonski, 2012).

La consecución de estas teorías y concepciones sobre la inteligencia del ser humano suponen un cambio significativo en el modelo educativo, ya que implica la toma en consideración de las potencialidades innatas del alumnado, transformando la enseñanza en un proceso de estimulación de cada una de las inteligencias o capacidades de los individuos.

\subsection{La competencia espacial y la Geografía}

En el ámbito de la educación, los trabajo sobre HE se han focalizado en las áreas de Ciencia, Tecnología, Ingeniería y Matemáticas (dominios STEM) (Wai, Lubinski \& Benbow, 2009) y, de forma especial en la geometría (McGee, 1979; Arrieta, 2006; Vázquez Maris y Noriega Biggio, 2010; Schelly, Anzolone, Wijnen \& Pearce, 2015) y 
en las ingenierías (Martín-Gutiérrez, García-Domínguez, Roca-González, Sanjuán-Hernán Pérez \& Mato-Carrodeguas, 2013; Melgosa Pedregosa, Ramos Barbero y Baños García, 2013; Torner, Alpiste y Brigos, 2014). La HE o CE es un tipo de inteligencia involucrada con objetos. Esta, a diferencia de la inteligencia lógico-matemática, que tiene una trayectoria de abstracción creciente, permanece ligada en lo fundamental al mundo concreto y de ahí su "poder de permanencia" (Gardner, 2006; Villa Sicilia, 2016, p. 43). Su consecución se encuentra ligada a la identificación de los componentes básicos que la configuran. En este sentido, existen diversas propuestas en relación a los elementos que la conforman. Así, McGee (1979) y Maier (1996) reconocen cinco componentes de la HE: 1) Percepción espacial o capacidad de localizar la posición horizontal o vertical de un objeto; 2) Visualización espacial o capacidad de visualizar la configuración de un objeto en estado dinámico (en movimiento o desplazamiento de sus partes); 3) Rotaciones mentales de figuras 2D y 3D; 4) Relaciones espaciales o capacidad de interpretar una composición así como las partes que lo conforman y la relación entre ellas y 5) Orientación espacial de uno mismo física o mentalmente en el espacio (Villa Sicilia, 2016).

Junto a tales capacidades se encuentra otra variante de la orientación espacial, que implica la capacidad de situar, mentalmente, un elemento en el espacio y llegar a previsualizarlo (pensarlo) desde diferentes perspectivas (Hegarty \& Waller, 2005). Dicha capacidad está íntimamente unida a la disciplina geográfica.

La actual normativa, que concierne a la educación en España, define la materia "Geografía" como "forma de conocimiento que estudia la diferenciación del espacio geográfico y la interacción entre la sociedad y el medio natural, entendida como un proceso geográfico en el que se manifiestan las estrategias, intereses y proyectos de las personas y de los grupos sociales en un determinado espacio" (Real Decreto 1105/2014, de 26 de diciembre).

Estos aprendizajes promueven el conocimiento de: "la distribución, localización e interconexión de los diferentes elementos que caracterizan los lugares y los espacios geográficos de diferente magnitud, de las sociedades que lo habitan y su diversidad cultural, del uso que hacen de los recursos del medio natural y de la organización del territorio" (Real Decreto 1105/2014, de 26 de diciembre).

Para el caso concreto de Educación Primaria, en el bloque 2: El mundo en que vivimos, encontramos algunos de los contenidos referentes a la geografía. En ellos, se señala como clave "La orientación espacial" (Real Decreto 126/2014 de 28 de febrero, 19375); apareciendo recogida en los siguientes Criterios de Evaluación y Estándares de aprendizaje:

\section{Criterios de Evaluación}

- No3: "Localizar"

- $\quad N^{0} 5$. "Explicar las distintas formas de representar la superficie terrestre."

- $\quad N^{0} 6$. "Describir correctamente planos y mapas interpretando su escala y signos convencionales."

- N07. "Identificar y manejar los conceptos de paralelos, meridianos y coordenadas geográficas."

- $\quad$ N015. "Describir las características del relieve de España y su red hidrográfica, localizándolos en un mapa."

- $\quad$ №16. "Identificar las principales unidades del relieve de Europa sus climas y su red hidrográfica, localizándolos en un mapa."

- Estándares de aprendizaje:

- N05.1. Explica las distintas representaciones de la Tierra, planos, mapas, planisferios y globos terráqueos.

- No6.1. Identifica y clasifica los diferentes tipos de mapas, incluyendo los planisferios, define qué es la escala en un mapa y utiliza e interpreta los signos convencionales más usuales que pueden aparecer en él.

- N07.1. Localiza diferentes puntos de la Tierra empleando los paralelos y meridianos y las coordenadas geográficas.

- $\quad$ №15.1. Localiza en un mapa las principales unidades del relieve de España y sus vertientes hidrográficas.

- N N15.2. Sitúa en un mapa los mares, océanos y los grandes ríos de España.

- $\quad$ N¹6.1. Localiza en un mapa el relieve de Europa, sus vertientes hidrográficas y sus climas. 
La CE comporta un papel esencial en el desarrollo de nuevos conocimientos. En este sentido, mejora la capacidad mental para la generación y comprensión de imágenes visuales (Kell, Lubiski, Benbow \& Steiger, 2013); fomenta el desarrollo del pensamiento verbal como habilidad clave para una correcta evolución de las capacidades cognitivas (Carroll, 1993) y ayuda a razonar en campos que, aparentemente, no están vinculados con la inteligencia espacial como la comprensión y confección de mapas mentales, esquemas, gráficos o croquis (Ontoria, 2006), entre otros aspectos.

\subsection{La competencia espacial y las tecnologías}

En el contexto actual, donde los requerimientos para la formación de la ciudadanía de la SIC están generando transformaciones en el ámbito de la educación, las universidades del Espacio Europeo de Educación Superior (EEES) se afanan por implementar modificaciones en los modelos y las metodologías de enseñanza y aprendizaje (E-A). El objetivo no es otro que la consecución de un sistema educativo superior adaptado a las nuevas necesidades en el ámbito de la tecnología, dotando a los centros y departamentos de recursos y de conectividad a Internet; prestando especial atención al rol del profesorado y a su competencia digital pues son los docentes quienes, en este contexto, adquieren un papel central para la correcta incorporación de los nuevos recursos TIC, transformándose en Tecnologías del Aprendizaje y del Conocimiento (TAC) (Gómez-Trigueros, 2019; Gómez-Trigueros y Binimelis, 2020).

Los recursos tecnológicos pueden ayudar en el aprendizaje y la comprensión del espacio de una forma autónoma (Jones, Ramanau, Cross \& Healing, 2010; Gonzato y Godino, 2013; Fombona Cadavieco, 2014; Giraldo Restrepo, 2015; Fombona Cadavieco y Vázquez-Cano, 2017). Como ya se ha señalado, para el caso concreto de la ciencia geográfica, el conocimiento espacial es imprescindible pues este contenido estructurante forma parte de su esencia; la distribución de los fenómenos en el territorio ha sido el objeto de estudio, a lo largo del tiempo, de la geografía, constituyendo el principal objetivo en la enseñanza escolar reglada. Las herramientas TIG (Tecnología de Información Geográfica) y su aplicación didáctica pueden llegar a desarrollar programas educativos que permitan una formación integral del alumnado puesto que permiten acercar el medio físico al discente.

A tenor de todo lo anterior, se considera necesario, para ayudar a la capacitación de los futuros docentes, llevar a cabo el análisis sobre la percepción de una muestra compuesta por profesorado en formación ( $n=383$ ), en relación a su CDD, CE y compararla con la valoración que realizan de una práctica de aula con tecnología, para la consecución de dichas competencias. Es por ello que el propósito de esta investigación se ha centrado en el trabajo, a través de diferentes hardware (dispositivos móviles) y de software (de geolocalización), de la adquisición y desarrollo de la CE y la CDD en la formación de los futuros docentes. Todo ello, sustentado en el modelo de E-A TAPCK, validado con uno de los más adecuados para la correcta inclusión de la tecnología en los procesos educativos. Se han extraído conclusiones que, aunque no definitivas, aportan importantes reflexiones para futuras investigaciones sobre el tema.

\section{METODOLOGÍA}

\subsection{Diseño de la investigación}

El trabajo se ha planteado desde un enfoque descriptivo, con una metodología mixta (Sánchez-Gómez, Rodrigues y Costa, 2018). Para su desarrollo se ha empleado un diseño de investigación de tipo exploratorio, basado en el uso del cuestionario como instrumento de recogida de información (Pardo, Ruiz y San-Martín, 2015; Gómez-Trigueros y Binimelis, 2020). La investigación se ha desarrollado durante el curso académico 2019-2020, en el contexto de aprendizaje de una Facultad de Educación de una universidad española. El proceso del estudio se ha configurado a lo largo de cuatro fases (Gómez Trigueros, 2015): revisión teórica sobre las CDD, el modelo TPACK, las TIG y estudios desarrollados previamente sobre el concepto de competencia 
espacial-geoespacial; el diseño y la posterior validación de los instrumentos a partir de la colaboración de profesorado de otras universidades nacionales e internacionales; la recogida de información a través del cuestionario 1 y el desarrollo de la práctica de aula y, por último, la distribución y vaciado de información del cuestionario 2.

Por la limitación en la extensión de palabras, así como el proceso investigativo, en este artículo se muestran los resultados cuantitativos del trabajo, descriptivos y concluyentes de los objetivos propuestos.

\subsection{Muestra}

La muestra objeto del estudio se ha seleccionado de manera no probabilística, dirigida e intencional (Argibay, 2009); consta de 383 participantes, docentes en formación, de Grado y de Postgrado, procedentes de instituciones universitarias españolas. La muestra se considera significativa respecto del total de la población existente (Buendía, Colás y Hernández, 1998) y se compone de 289 mujeres $(75,4 \%)$ y 94 hombres $(24,6 \%)$. El rango de edades está comprendido entre los 19 años y más de 40 años.

\subsection{Instrumentos}

En esta investigación, se ha optado por un diseño metodológico basado en los estudios tipo encuesta, y una metodología cuantitativa transversal de corte descriptivo e inferencial. De un lado, se han aplicado el cuestionario 1, adaptado a los objetivos del estudio y diseñado ad hoc, a partir del empleado por Schmidt et al., (2009) y Gómez Trigueros (2015), cuyo contenido ha sido validado por expertos de universidades públicas españolas e internacionales, para el presente estudio. Consta de 15 ítems medidos en una escala Likert de cinco puntos (1, Muy en desacuerdo - 5, Totalmente de acuerdo), y organizados en cinco dimensiones de estudio: 1. Características sociodemográficas (ítems 1-3); 2. Conocimiento del Contenido (CK) (ítems 4-6); 3. Conocimiento Tecnológico Pedagógico (TPK) (7-9); 4. Conocimiento Tecnológico del Contenido (TCK) (ítems 10-12); y 5. Conocimiento Pedagógico y de Contenido (PCK) (ítems 13-15) (tabla 1).

De otro lado, el cuestionario 2, igualmente validado y conformado a partir de las mismas dimensiones, con una redacción distinta para no condicionar las respuestas ni proporcionar un adiestramiento de los participantes (tabla 1) (Gargallo-López et al., 2017) y con la intencionalidad de lograr un análisis diacrónico o longitudinal que implica el estudio de uno o varios fenómenos a lo largo del tiempo como es el caso (López-Roldán y Fachelli, 2015).

Con el fin de verificar la fiabilidad de los cuestionarios, se ha calculado el coeficiente alpha de Cronbach (Raykov \& Marcoulides, 2017). Los resultados obtenidos (cuestionario $1 a=.903$; cuestionario $2 a=.958$ ) constatan la existencia de una alta y adecuada consistencia interna de ambos instrumentos para el estudio propuesto (Hernández, Fernández y Baptista, 2003; Bisquerra Alzina, 2004). Del mismo modo, se hallado el índice Chi-Cuadrado de Pearson con resultados de p-valor $<1=$ Sig. 0.001 en ambos instrumentos (Cohen, Manion \& Morrison, 2008), indicativo de la alta correlación de las preguntas planteadas ilustrativo de la validez de los ítems y la estructura de los instrumentos implementados.

\subsection{Procedimiento}

En relación al procedimiento, se distribuyeron dos cuestionarios, uno anterior a la intervención (cuestionario 1) y otro después de trabajar con los participantes los contenidos y las competencias geoespaciales (cuestionario 2) (tabla 1). Se facilitaron por correo electrónico a través de la aplicación gratuita Google Forms, durante el segundo cuatrimestre del curso académico analizado (del 20 de septiembre al 18 de diciembre de 2019). Los estudiantes recibieron sendos cuestionarios a través de su correo institucional de la universidad y se les informó del objetivo de la investigación, así como de la confidencialidad de las respuestas. 
Tabla 1. Cuestionarios de la investigación (cuestionario 1; cuestionario 2).

\begin{tabular}{|c|c|c|}
\hline \multicolumn{3}{|r|}{ Cuestionario 1} \\
\hline D. & Var. & Ítem \\
\hline \multirow{3}{*}{ 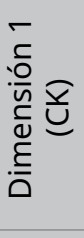 } & $\mathrm{CK}_{1}$ & Soy capaz de definir el concepto “orientación” geográfica. \\
\hline & $\mathrm{CK}_{2}$ & $\begin{array}{l}\text { Cuento con habilidades y destrezas para poder orientarme en el espacio geográfico a partir de los } \\
\text { puntos cardinales y coordenadas cartográficas. }\end{array}$ \\
\hline & $\mathrm{CK}_{3}$ & Soy capaz de localizar elementos del espacio geográfico en un mapa y/o plano. \\
\hline \multirow{3}{*}{ 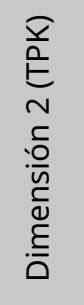 } & $\mathrm{TPK}_{1}$ & $\begin{array}{l}\text { Conozco recursos TIG y tecnologías adecuadas para optimizar la enseñanza de la Geografía en } \\
\text { relación a localizar un lugar y orientarse. }\end{array}$ \\
\hline & $\mathrm{TPK}_{2}$ & $\begin{array}{l}\text { Soy capaz de seleccionar las TIG apropiadas para optimizar la enseñanza de los contenidos y com- } \\
\text { petencias relativas a la geolocalización. }\end{array}$ \\
\hline & $\mathrm{TPK}_{3}$ & $\begin{array}{l}\text { La implementación de las TIG en la E-A de la Geografía capacitan para usar las tecnologías en acti- } \\
\text { vidades de enseñanza de las competencias geoespaciales. }\end{array}$ \\
\hline \multirow{3}{*}{ 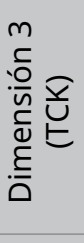 } & $\mathrm{TCK}_{1}$ & Sé usar software específico sobre contenidos geográficos relativos a la localización espacial. \\
\hline & $\mathrm{TCK}_{2}$ & $\begin{array}{l}\text { Conozco qué tecnologías de la información se pueden aplicar para enseñar cómo orientarse en el } \\
\text { espacio. }\end{array}$ \\
\hline & $\mathrm{TCK}_{3}$ & Soy capaz de usar software y hardware específico para el diseño de actividades de geolocalización. \\
\hline \multirow{3}{*}{ 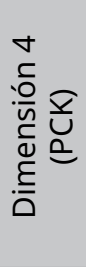 } & $\mathrm{PCK}_{1}$ & Soy capaz de resolver dificultades didácticas relacionadas con las competencias geoespaciales. \\
\hline & $\mathrm{PCK}_{2}$ & $\begin{array}{l}\text { Estoy capacitado/a para formular objetivos didácticos específicos del currículo de Geografía relati- } \\
\text { vos a las competencias geoespaciales. }\end{array}$ \\
\hline & $\mathrm{PCK}_{3}$ & $\begin{array}{l}\text { Estoy habilitado/a para seleccionar herramientas TIG adecuadas de evaluación del desempeño de } \\
\text { los estudiantes en el aprendizaje de las competencias geoespaciales. }\end{array}$ \\
\hline \multicolumn{3}{|r|}{ Cuestionario 2} \\
\hline D. & Var. & Ítem \\
\hline \multirow{3}{*}{ 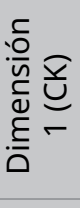 } & $\mathrm{CK}_{1}$ & Soy capaz de identificar y de manejar los conceptos de paralelos, meridianos. \\
\hline & $\mathrm{CK}_{2}$ & Sé orientarme en el espacio a partir de coordenadas cartográficas proporcionadas. \\
\hline & $\mathrm{CK}_{3}$ & Sé localizar un punto en un plano, un mapa. \\
\hline \multirow{3}{*}{$\begin{array}{l}\frac{a}{a} \\
\text { E } \\
N \\
\frac{c}{0} \\
: \frac{0}{n} \\
\frac{n}{d} \\
\frac{E}{0} \\
\overline{0}\end{array}$} & $\mathrm{TPK}_{1}$ & $\begin{array}{l}\text { Los recursos TIG utilizados y las prácticas en las que he participado me permiten elegir las tecno- } \\
\text { logías adecuadas para optimizar la enseñanza de la Geografía, como el uso de Google Earth, para } \\
\text { localizar un lugar y orientarme. }\end{array}$ \\
\hline & $\mathrm{TPK}_{2}$ & $\begin{array}{l}\text { Los recursos TIG y los contenidos trabajados en mi formación me han preparado para usar las } \\
\text { tecnologías de la información para mejorar la motivación del alumnado para aprender conteni- } \\
\text { dos tales como orientación y localización espacial. }\end{array}$ \\
\hline & $\mathrm{TPK}_{3}$ & $\begin{array}{l}\text { Las TIG como Google Earth ayudan a utilizar las tecnologías para la promover la participación } \\
\text { activa del alumnado en las actividades relacionadas con la geolocalización. }\end{array}$ \\
\hline \multirow{3}{*}{ 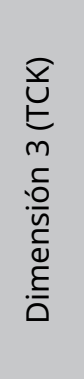 } & $\mathrm{TCK}_{1}$ & $\begin{array}{l}\text { La formación y las prácticas en las que he participado me han enseñado qué tecnologías de la } \\
\text { información se pueden aplicar para enseñar cómo orientarse en el espacio. }\end{array}$ \\
\hline & $\mathrm{TCK}_{2}$ & $\begin{array}{l}\text { Las actividades realizadas sobre localización favorecen la adquisición de competencias geoespa- } \\
\text { ciales para la selección de tecnologías de la información aplicables a la enseñanza y aprendizaje } \\
\text { de la Geografía. }\end{array}$ \\
\hline & $\mathrm{TCK}_{3}$ & $\begin{array}{l}\text { La formación recibida y las prácticas en las que he participado enseñan a usar las TIG de manera } \\
\text { adecuada para la enseñanza de contenidos geográficos relativos a la orientación y localización } \\
\text { espacial, como los recursos multimedia, el software de simulación, etc. }\end{array}$ \\
\hline
\end{tabular}




\begin{tabular}{|c|c|c|}
\hline \multicolumn{3}{|r|}{ Cuestionario 2} \\
\hline D. & Var. & Ítem \\
\hline \multirow{3}{*}{ 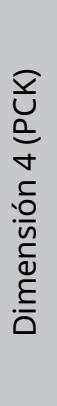 } & $\mathrm{PCK}_{1}$ & $\begin{array}{l}\text { La formación recibida y las prácticas en las que he participado me han formado para guiar a los } \\
\text { estudiantes en la realización de actividades en las que desarrollar sus habilidades y destrezas de } \\
\text { geolocalización. }\end{array}$ \\
\hline & $\mathrm{PCK}_{2}$ & $\begin{array}{l}\text { La formación recibida y las prácticas en las que he participado habilitan para poder seleccionar } \\
\text { herramientas TIG adecuadas de evaluación del desempeño de los estudiantes en el aprendizaje } \\
\text { de los contenidos de Geografía relativos a localización y orientación espacial. }\end{array}$ \\
\hline & $\mathrm{PCK}_{3}$ & $\begin{array}{l}\text { La formación recibida y las prácticas en las que he participado preparan para determinar qué } \\
\text { conceptos geográficos deben trabajarse en el currículo escolar para la consecución de las compe- } \\
\text { tencias geoespaciales. }\end{array}$ \\
\hline
\end{tabular}

Fuente: Elaboración propia.

Respecto a la propuesta didáctica, se diseñó una práctica grupal, fuera del aula, orientada al trabajo de la CE. La actividad a desarrollar consistió en la geolocalización de hitos artístico-culturales relevantes plasmados en ocho rutas del Campus de la Universidad. Se conformaron grupos de 3-5 estudiantes a los que se les proporcionó una de las rutas. Asimismo, se distribuyeron las indicaciones relativas al material necesario para el desarrollo de las tareas y otros aspectos a tener en cuenta al realizarse en un contexto distinto al aula; se explicó, con detalle, el proceso a seguir para llevar a cabo un uso correcto de las TIG (dispositivos móviles; confección de QR; programas de geolocalización) así como el formato de entrega de la tarea final. Esta información se transmitió a todos los participantes previamente a la realización de la práctica y se colgó en el espacio de Campus Virtual disponible para la asignatura de "Didáctica de la CC. Sociales: Geografía", en la Universidad. Los resultados, cualitativos de dichas actividades no se reseñan en este trabajo por la limitación espacial de palabras y porque no alteran las conclusiones a que se llega con los instrumentos (cuestionarios 1 y 2 ) implementados.

Una vez recopilada la información de los grupos, se ha realizado el análisis de los datos cuantitativos mediante el paquete estadístico SPSS v.25. Teniendo en cuenta los objetivos propuestos, se han realizado diferentes pruebas. En una fase inicial, se valoró la consistencia interna del cuestionario y se obtuvieron los principales estadísticos descriptivos del conjunto de respuestas cuantitativas (media=M y desviación típica=DT). Tras ello, se realizó también un análisis exploratorio de los estadísticos descriptivos de prueba a tenor de las dimensiones que conforman el instrumento. En una segunda fase, se aplicó la prueba $t$ de Student para muestras independientes, para comprobar si existían diferencias significativas antes y después de la intervención.

\section{RESULTADOS}

\subsection{Análisis descriptivos}

En este apartado, se presenta una selección de los resultados obtenidos en este estudio, limitado a los resultados de los cuestionarios implementados, comenzando por el análisis descriptivo de las puntuaciones de los participantes considerando los ítems que los conforman.

La comparativa de los resultados de los estadísticos descriptivos (media=M; desviación típica=DT) de cada una de las dimensiones (tabla 2) subraya la escasa o nula CE de los participantes previo a la intervención.

En la dimensión CK (ítems 4-6), que arroja información sobre la percepción del alumnado en relación a sus conocimientos de geolocalización, es donde los ítems presentan las diferencias más significativas entre el cuestionario $1(M \geq 2.03 ; D T \leq 0.658)$ y $2(M \geq 4.88 ; D T \leq 0.573)$. Estos valores indican la escasa formación previa de los participantes sobre contenidos como: localización, orientación, paralelos, meridianos, etc. 
Tabla 2. Resultados descriptivos (media=M; desviación típica=DT) cuestionario 1 y cuestionario 2.

\begin{tabular}{|c|c|c|c|c|c|}
\hline \multirow{2}{*}{ Ítem } & & \multicolumn{2}{|c|}{ Cuestionario 1} & \multicolumn{2}{|c|}{ Cuestionario 2} \\
\hline & & M & DT & M & DT \\
\hline \multicolumn{6}{|c|}{ Dimensión 1 Conocimiento del Contenido (CK) } \\
\hline Ítem 4 & $\mathrm{CK}_{1}$ & 2.03 & 0.665 & 4.92 & 0.573 \\
\hline Ítem 5 & $\mathrm{CK}_{2}$ & 2.65 & 0.634 & 4.91 & 0.523 \\
\hline Ítem 6 & $\mathrm{CK}_{3}$ & 2.72 & 0.658 & 4.88 & 0.545 \\
\hline \multicolumn{6}{|c|}{ Dimensión 2 Conocimiento Tecnológico Pedagógico (TPK) } \\
\hline Ítem 7 & $\mathrm{TPK}_{1}$ & 3.41 & 0.610 & 4.97 & 0.627 \\
\hline Ítem 8 & $\mathrm{TPK}_{2}$ & 3.71 & 0.695 & 4.78 & 0.644 \\
\hline Ítem 9 & $\mathrm{TPK}_{3}$ & 3.35 & 0.682 & 4.91 & 0.624 \\
\hline \multicolumn{6}{|c|}{ Dimensión 3 Conocimiento Tecnológico del Contenido (TCK) } \\
\hline Ítem 10 & $\mathrm{TCK}_{1}$ & 3.04 & 0.596 & 4.84 & 0.513 \\
\hline Ítem 11 & $\mathrm{TCK}_{2}$ & 3.13 & 0.537 & 4.94 & 0.438 \\
\hline Ítem 12 & $\mathrm{TCK}_{3}$ & 3.24 & 0.548 & 4.69 & 0.512 \\
\hline \multicolumn{6}{|c|}{ Dimensión 4 Conocimiento Pedagógico y de Contenido (PCK) } \\
\hline Ítem 13 & $\mathrm{PCK}_{1}$ & 2.67 & 0.671 & 4.85 & 0.598 \\
\hline Ítem 14 & $\mathrm{PCK}_{2}$ & 2.12 & 0.683 & 4.83 & 0.577 \\
\hline Ítem 15 & $\mathrm{PCK}_{3}$ & 2.18 & 0.693 & 4.96 & 0.582 \\
\hline
\end{tabular}

Fuente: Elaboración propia.

Respecto a la dimensión TPK (ítems 7-9), los valores muestran la percepción negativa de los participantes, previa al trabajo de los contenidos, en relación a su capacidad para comprender y reconocer los recursos TIG y otras tecnologías adecuadas para el desarrollo de las competencias espaciales en su labor docente $(M \geq 3.35 ; \mathrm{DT} \leq 0.695)$ (cuestionario 1). Por su parte, esta misma dimensión arroja valores positivos, que se aproximan a la opción de respuesta "Totalmente de acuerdo" ( $\mathrm{M} \geq 4.78 ; \mathrm{DT} \leq 0.644)$ a lo largo del cuestionario 2. Lo mismo sucede respecto de la dimensión TCK (ítems 10-12), donde la muestra considera que, antes de la intervención, no tiene suficiente conocimiento sobre las posibilidades pedagógicas y las limitaciones en el manejo de software y hardware para trabajar la geolocalización y para capacitar en competencias geoespaciales ( $\mathrm{M} \geq 3.04$; $\mathrm{DT} \leq 0.596)$ (cuestionario 1). Una vez impartidos los contenidos y trabajada la CE con TPACK, los valores de respuestas (cuestionario 2) se acercan a la opción "Totalmente de acuerdo" $(\mathrm{M} \geq 4.69 ; \mathrm{DT} \leq 0.513)$.

También, se observa esta mista tendencia en la dimensión PCK (ítems 13-15), con valores de respuesta en el cuestionario 1 próximos a "En desacuerdo" ( $M \geq 2.12$; $\mathrm{DT} \leq 0.693)$, indicativos de la percepción de la muestra en relación a su potencial para formular objetivos didácticos, procesos de evaluación o resolución de dudas sobre competencias geoespaciales. De igual forma, tras la práctica docente, los resultados para esta misma dimensión son equivalentes al valor 5 de la escala Likert $(M \geq 4.81 ; D T \leq 0.598)$ (cuestionario 2). 


\subsection{Análisis paramétricos}

Para determinar si existen diferencias significativas entre las dimensiones analizadas y atendiendo a las diferencias que se han podido apreciar en los resultados de los descriptivos (M y DT), se ha procedido a comparar, mediante estadística inferencial, las puntuaciones obtenidas en las variables de cada fase (cuestionario 1 y 2). Para ello, aplicamos la prueba $t$ de Student para muestras relacionadas (tabla 3), donde hemos incorporado también la información respecto a la comprobación de los supuestos paramétricos de homocedasticidad y normalidad. A este respecto, indicar que el valor de la homocedasticidad de las variables arroja valores compatibles con la aplicación de pruebas paramétricas. En cuanto a la normalidad, la prueba de Kolmogorov-Smirnov (con corrección de significatividad de Lillierfors), el nivel de significación es superior a 0.05 ( $p \geq 0.09$ ), ajustándose a la normalidad con lo que sí se puede aplicar la prueba paramétrica $t$ de Student para muestras relacionadas.

Por lo que respecta a los resultados de los supuestos paramétricos, podemos observar la existencia de una significatividad estadística en todas las variables respecto a las diferencias entre ambas fases del estudio (cuestionario 1 y 2). Esto denota la valoración positiva respecto de las posibilidades didácticas de la intervención realizada en las diferentes dimensiones que han sido consideradas para el estudio.

Tabla 3. Resultados de los supuestos paramétricos y del análisis correlacional (prueba t de Student).

\begin{tabular}{|c|c|c|c|c|c|}
\hline \multirow{2}{*}{ Dimensión } & \multirow{2}{*}{ Cuestionario } & \multicolumn{2}{|c|}{ t de Student } & \multicolumn{2}{|c|}{ Correlación muestras } \\
\hline & & $\mathrm{t}$ & Sig. unilateral & Correlación & Sig. \\
\hline \multirow{2}{*}{ CK } & 1 & \multirow{2}{*}{-2.765} & \multirow{2}{*}{0.007} & \multirow{2}{*}{0.816} & \multirow{2}{*}{0.004} \\
\hline & 2 & & & & \\
\hline \multirow{2}{*}{ TPK } & 1 & \multirow{2}{*}{-4.056} & \multirow{2}{*}{0.021} & \multirow{2}{*}{0.799} & \multirow{2}{*}{0.043} \\
\hline & 2 & & & & \\
\hline \multirow{2}{*}{ TCK } & 1 & \multirow{2}{*}{-3.029} & \multirow{2}{*}{0.010} & \multirow{2}{*}{0.812} & \multirow{2}{*}{0.001} \\
\hline & 2 & & & & \\
\hline \multirow{2}{*}{ PCK } & 1 & \multirow{2}{*}{-2.369} & \multirow{2}{*}{0.009} & \multirow{2}{*}{0.834} & \multirow{2}{*}{0.005} \\
\hline & 2 & & & & \\
\hline
\end{tabular}

Fuente: Elaboración propia.

No obstante, aunque a nivel estadístico se presenten diferencias significativas en todas las variables dependientes, la dimensión TPK ha sido el área donde es mayor la distancia en las valoraciones dadas por el alumnado participante.

Destacar el valor $t$ para la dimensión CK con un valor de significación unilateral de $0.007(\leq 0.05)$ así como una correlación significativa ( $p=0.004)$ entre las variables dependientes de dicha dimensión.

\section{DISCUSIÓN Y CONCLUSIONES}

Las competencias geospaciales son un conocimiento clave para la ciudadanía como así aparece recogido en las actuales normativas educativas nacionales (Ley Orgánica 8, 2013) e internacionales (OCDE, 2010; UNESCO, 2016; INTEF, 2017). Es labor de los docentes proveer al alumnado de tales competencias para que puedan contar con una formación plena, que les permita relacionarse en el contexto educativo y laboral del siglo XXI (Delors, 1996). El potencial de la información geoespacial y su interés para la ciudadanía está 
vinculado, de manera íntima, con la toma de decisiones y la gestión (Muro-Medrano, 2012), y también para el desenvolvimiento profesional (Tsou \& Yanow, 2010).

Junto a las habilidades geoespaciales, la capacitación en el uso, manipulativo, crítico e informativo de las tecnologías resulta un elemento fundamental en el desarrollo de los actuales procesos de E-A. En este contexto, el docente debe poseer una serie de conocimientos con los cuales pueda desarrollar una eficaz y eficiente integración educativa de las tecnologías (Campos \& Solano, 2017); dirigidos hacia sus prácticas docentes; teniendo en cuenta las teorías en las que se apoya la pedagogía sin descuidar un profundo conocimiento de los contenidos propios de su disciplina. La complementariedad de todos estos requerimientos son la base del modelo de E-A TPACK (Gómez-Trigueros, 2019; Ortega-Sánchez y Gómez-Trigueros, 2019). A partir de la interrelación de las dimensiones del modelo (los conocimientos disciplinares (CK); pedagógicos (PK) y tecnológicos (TK) y sus combinaciones (PCK, TCK, TPK) se procura que el profesorado construya entornos de aprendizaje enriquecidos, en los cuales se favorezca la consecución de los objetivos educativos planteados.

Es necesario, pues, abordar la correcta formación de los futuros docentes para que cuenten con CDD y, para el caso concreto de las Ciencia Sociales, con CE (Gómez-Trigueros y Binimelis, 2020). Es por ello que este estudio investiga la situación actual con respecto a los conocimientos que poseen los futuros docentes en tres dimensiones del modelo TPACK: conocimientos disciplinares sobre competencia geoespacial del profesorado en formación antes y después del curso (CK); conocimiento sobre su competencia digital docente (TCK) y su percepción sobre su importancia manipulativa, didáctica y pedagógica (TPK y PCK).

Los resultados obtenidos en esta investigación evidencian que los docentes, participantes en el estudio, cuenta con una deficiente competencia geoespacial (dimensión CK) cuando inician su preparación como futuros profesores ( $2^{\circ}$ curso de Grado y Máster de Profesorado). Una vez implementadas estrategias y actividades orientadas al desarrollo de su CE con TIG en su formación esta capacitación mejora sustancialmente, proveyendo a los futuros docentes de tales competencias geoespaciales como se confirma en este trabajo. Tales resultados convergen con las investigaciones de otros autores, en relación a estudios superiores de corte técnico (Vázquez Maris y Noriega Biggio, 2010; Vázquez Maris, Noriega Biggio y Maris García, 2013) y humanidades (Llancavil Llancavil \& González Vega, 2017; Cebolla Martínez, Gómez Cabello y López Martín, 2017).

Del mismo modo, se detecta una escasa capacitación de la muestra en metodologías que combinen, adecuadamente, contenidos y tecnologías (dimensión PCK). Por el contrario, y dando respuesta a otro de los objetivos planteados en este trabajo, los valores obtenidos muestran una percepción positiva de los estudiantes sobre su capacidad profesional en competencias digitales (dimensión TPK) y en el uso de las tecnologías para la enseñanza de contenidos geoespaciales (dimensión TCK). Conclusiones similares se alcanzaron por otros investigadores (Roig-Vila et al., 2015; Gómez-Trigueros, 2019) quienes confirman que los docentes en formación no presentan dificultades para la manipulación de dispositivos digitales. Esta percepción puede deberse a que el futuro profesorado tiende a interpretar el uso manipulativo de las tecnologías y su capacitación digital como docente en dos ámbitos distintos, desvinculando las competencias propiamente docentes de las competencias digitales del profesorado.

Otro objetivo del estudio ha sido el análisis de la capacidad de los docentes noveles para seleccionar recursos tecnológicos adecuados a unos contenidos concretos. Así, el estudio presenta una deficiente capacidad para discernir cómo implementar, adecuadamente, los recursos TIC y TIG, para la transmisión de los contenidos relativos a la localización y la orientación (dimensión PCK) previamente a la intervención. Tras la formación recibida, se confirma una clara mejoría en relación a la selección adecuada de herramientas TIG para el trabajo de los contenidos sobre geolocalización y se acredita la cualificación de los participantes para implementar las tecnologías en el aula (TCK). De igual forma, se corrobora el valor que, el profesorado en formación otorga a dicha capacitación. Estos hallazgos coinciden con otros estudios (Emine, Emre \& Kamil, 2014; Gómez-Trigueros, Ruiz-Bañuls y Ortega-Sánchez, 2019; Gómez-Trigueros y Binimelis, 2020), donde se afirma la necesidad de una formación digital del profesorado y sus beneficios para la docencia, así como la importancia de la adecuación de los planes de estudio a tales requisitos de la SIC (Mishra \& Koehler, 2006; Roig-Vila et al., 2015; Cabero Almenara y Ruiz Palmero, 2017; Ortega-Sánchez y Gómez-Trigueros, 2019). 
Todas estas evidencias nos permiten concluir que, a pesar de la importancia de enseñar la capacidad espacial a la ciudadanía, para lograr asociar y relacionar contenidos que en un principio pueden no tener relación directa, los currículos de Grado de Maestro/a en Educación Primaria, en el área de Ciencias Sociales: Geografía y de Máster de Profesorado, especialidad Geografía e Historia, continúan sin apostar por prácticas de aula que atiendan, de manera concreta, tales requerimientos. De ahí que se quieran mostrar tales carencias en este trabajo. Asimismo, y a pesar de la positiva autoeficacia en competencias digitales del futuro profesorado, se constata la falta de adecuación de las propuestas curriculares de Grado y Postgrado del profesorado para la correcta implementación de las TIG en la formación inicial. En este sentido, aunque las administraciones educativas están llevando a cabo propuestas para la mejora en la formación e implementación de las tecnologías en los planes de estudio universitarios (Gómez-Trigueros y Binimelis, 2020), todavía queda un largo camino para dar cumplimiento a las actuales demandas de la SIC. Es imprescindible, para la mejora educativa y la comprensión del espacio geográfico, potenciar las habilidades relacionadas con la competencia digital como la recuperación, selección, creación o intercambio de contenidos digitales y de experiencias en entornos virtuales (Martínez-Bravo, Sádaba y Serrano-Puche, 2018; Colás-Bravo, Conde-Jiménez y Reyes-de-Cózar, 2019; Gómez-Trigueros, 2019); y con las competencias geoespaciales.

\section{FINANCIACIÓN}

Esta investigación ha sido realizada gracias a la financiación de los Proyectos de Innovación Educativa de la Universidad de Alicante REDESI3CE2020-4612.

\section{REFERENCIAS}

Argibay, J.C. (2009). Muestra en investigación cuantitativa. Subjetividad y Procesos Cognitivos, 13(1), 13-29. Recuperado de http://www.redalyc.org/articulo.oa?id=339630252001.

Arrieta, M. (2006). La capacidad espacial en la educación matemática: estructura y medida. Educación Matemática, 18(1), 99-132.

Braslavsky, C. y Acosta, F. (2006). La Formación en Competencias para la Gestión de la Política Educativa: un Desafío para la Educación Superior en América Latina. REICE. Revista Iberoamericana sobre Calidad, Eficacia y Cambio en Educación, 4(2), 27-42.

Bisquerra Alzina, R. (2004). Metodología de la investigación educativa. Madrid: Arco Libros-La Muralla.

Buendía, L., Colás, M.P. y Hernández, F. (1998). Métodos de investigación en Psicopedagogía. Madrid: McGraw-Hill.

Cabero Almenara, J. y Ruiz Palmero, J. (2017). Las Tecnologías de la Información y Comunicación para la inclusión: reformulando la brecha digital. Ijeri. International Journal of Educational Research and Innovation, 9, 16-30. Recuperado de https://www.upo.es/revistas/index.php/IJERI/article/view/2665

Cabero Almenara, J., Roig-Vila, R. y Mengual-Andrés, S. (2017). Conocimientos tecnológicos, pedagógicos y disciplinares de los futuros docentes según el modelo TPACK. Digital Education Review, 32, 73-84. https://doi.org/10.1344/ der.2017.32.73-84

Campos, J. \& Solano, W. (2017). The future of the teaching profession from the perspective of students with a Major in Education. Journal of New Approaches in Educational Research, 6(2), 87-92. https://doi.org/10.7821/naer.2017.7.191

Carroll, J. B. (1993). Human cognitive abilities: A survey of factor-analytic studies. Nueva York: Cambridge University Press. https://doi.org/10.1017/CBO9780511571312

Cebolla Martínez, R., Gómez Cabello, E., y López Martín, F. (2017). Aprendiendo Geografía con una IDE didáctica. Los geojuegos de IDEARAGON. Revista MAPPING, 26(182), 26-36.

Colás-Bravo, M.P., Conde-Jiménez, J. \& Reyes-Cózar, S. (2019). The development of the digital teaching competence from a sociocultural approach. Comunicar, 27(61). https://doi.org/10.3916/C61-2019-02

Cohen, L., Manion, L. \& Morrison, K. (2007). Research methods in education. London: Routledge. https://doi. org/10.4324/9780203029053 
Delors, J. (1996). La Educación Encierra un Tesoro. Madrid: Editorial Santillana, Ediciones UNESCO.

Dziekonski M. (2012). La inteligencia espacial: Una mirada a Howard Gardner. Arteoficio, 2, 7-12.

Emine, S., Emre, Ü. \& Kamil, İ. (2014). Primary School Second Grade Teachers' and Students' Opinions on Media Literacy. Comunicar, 21(42), 119-127. https://doi.org/10.3916/C42-2014-11

Fombona Cadavieco, J. (2014). La interactividad de los dispositivos móviles geolocalizados, una nueva relación entre personas y cosas. Historia y Comunicación Social, 18, 777-788. https://doi.org/10.5209/rev_HICS.2013.v18.44007

Fombona Cadavieco, J. y Vázquez-Cano, E. (2017). Posibilidades de utilización de la geolocalización y realidad aumentada en el ámbito educativo. Educación XX1, 20(2), 319-342. https://doi.org/10.5944/educxx1.19046

Gargallo-López, B., Pérez-Pérez, C., Verde-Peleato, I. y García-Félix, E. (2017). Estilos de aprendizaje en estudiantes universitarios y enseñanza centrada en el aprendizaje. RELIEVE, 23(2). https://doi.org/10.7203/relieve.23.2.9078

Gardner, H. (1983). Frames of mind: The theory of Multiple Iintelligences. New York: Basic Books.

Gardner, H. (1993). Frames of Mind. The Theory of Multiple Inteligencies. New York: Basic Books.

Gardner, H. (1999). Intelligence Reframed: Multiple Intelligences for the 21st Century. Nueva York: Basic Books.

Gardner, H. (2006). Multiple intelligences: New horizons in Theory and practice. (Rev. ed.). Basic Books.

Giraldo Restrepo, J.C. (2015). La cartografía en el ámbito de las Tecnologías de la Información y la Comunicación (TIC). Revista DIM, 31. Recuperado de http://www.pangea.org/dim/revista.htm.

Gómez Trigueros, I. M. (2015). Proyecto a partir del modelo TPACK para desarrollar el aprendizaje de la Geografía en los estudios de Grado de Educación Primaria (Tesis Doctoral). Universidad de Alicante, Alicante, España.

Gómez-Trigueros I.M., Ruiz-Bañuls M. \& Ortega-Sánchez D. (2019). Digital Literacy of Teachers in Training: Moving from ICTs (Information and Communication Technologies) to LKTs (Learning and Knowledge Technologies). Education Sciences, 9(4), 274. https://doi.org/10.3390/educsci9040274

Gómez-Trigueros, I.M. (2019). Methodologies Gamified as Didactic Resources for Social Sciences. International Journal of Emerging Technologies in Learning (iJET), 14(23), 193-207. https://doi.org/10.3991/ijet.v14i23.10794

Gómez-Trigueros, I.M. y Binimelis, J. (2020). Aprender y enseñar con la escala del mapa para el profesorado de la "generación Z": la competencia digital docente. Ar@cne: revista electrónica de recursos en internet sobre geografía y ciencias sociales, 1. https://doi.org/10.1344/ara2020.238.30561

Gonzato, M. y Godino, J. (2007). Habilidades de orientación espacial: de la cartografía al GPS. España: Universidad de Granada. Recuperado de http://www.ugr.es/ jmcontreras/thales/1/ComunicacionesPDF/HabilidadesOrientacion.pdf

Hegarty, M. \& Waller, D.A. (2005). Individual differences in spatial abilities. The Cambridge Handbook of Visuospatial Thinking. Cambridge: Cambridge University Press. https://doi.org/10.1017/CB09780511610448.005

Hernández, R., Fernández, C. y Baptista, M. P. (2010). Metodología de la investigación. México, D.F.: McGraw-Hill.

INTEF (2017). Marco Común de Competencia Digital Docente. Madrid: Instituto Nacional de Tecnologías Educativas y Formación del Profesorado. Madrid: Ministerio de Educación y Formación Profesional, octubre de 2017. Recuperado de http://cort.as/-GHoN>

Järvelä, S., Näykki, P., Laru, J. \& Luokkanen, T. (2007). Structuring and regulating collaborative learning in higher education with wireless networks and mobile tools. Educational Technology y Society, 10(4), 71 - 79.

Jones, C., Ramanau, R., Cross, S. \& Healing, G. (2010). Net generation or Digital Natives: Is there a distinct new generation entering university? Computers \& Education, 54(3), 722-732. https://doi.org/10.1016/j.compedu.2009.09.022

Kell, H.J., Lubinski, D., Benbow, C.P. \& Steiger, J.H. (2013). Creativity and technical innovation: Spatial ability's unique role. Psychological science, 24(9), 1831-1836. https://doi.org/10.1177/0956797613478615

Larsem-Freeman, D. (2000). Techniques and Principle in Language Teaching. Oxford: Oxford University Press.

Ley Orgánica 8/2013, de 9 de diciembre, para la Mejora de la Calidad Educativa (LOMCE), Boletín Oficial del Estado (BOE), núm. 295, martes 10 de diciembre de 2013, Sec. I. pág. 97858. Recuperado de http://www.boe.es/boe/ dias/2013/12/10/pdfs/BOE-A-2013-12886.pdf

Linn, M.C. \& Petersen, A.C., (1985). Emergence and characterization of sex differences in spatial ability: A meta-analysis. Child development, 1479-1498. https://doi.org/10.2307/1130467

Lohman, D. F. (1986). The effect of speed-accuracy tradeoff on sex differences in mental rotation. Perception and Psychophysics, 39(6), 427-436. https://doi.org/10.3758/BF03207071

Lohman, D. F. (1989). Human intelligence: An introduction to advances in theory and research. Review of Educational Research, 59(4), 333-373. https://doi.org/10.3102/00346543059004333 
López-Roldán, P. y Fachelli, S. (2015). Metodología de la investigación social cuantitativa. Barcelona: Universidad Autónoma de Barcelona.

Llancavil Llancavil, D. y González Vega, J. (2017). Un enfoque didáctico para la enseñanza del espacio geográfico. Revista Electrónica Diálogos Educativos, 14(28), 64-91. Recuperado de http://revistas.umce.cl/index.php/dialogoseducativos/article/view/1029

Maier, P.H. (1996). Spatial geometry and spatial ability-How to make solid geometry solid? In Elmar Cohors-Fresenborg, K. Reiss, G. Toener \& H.-G. Weigand (Ed.) Selected papers from the Annual Conference of Didactics of Mathematics, pp. 63-75. Osnabrueck.

Martín-Gutiérrez, J., García-Domínguez, M., Roca-González, C., Sanjuán-Hernán Pérez, A. \& Mato-Carrodeguas, C. (2013). Comparative Analysis Between Training Tools in Spatial Skills for Engineering Graphics Students Based in Virtual Reality, Augmented Reality and PDF3D Technologies. Procedia Computer Science, 25, 360-363. https://doi.org/10.1016/j.procs.2013.11.043

Martínez-Bravo, M.P., Sádaba, Ch. y Serrano-Puche, J. (2018). Desarrollo de competencias digitales en comunidades virtuales: un análisis de "ScolarTIC". Prisma Social. Revista de Ciencias Sociales e Investigación Social, 20, 129-159. Recuperado de http://revistaprismasocial.es/article/view/2318

Mateo, L. (2010). Origen y desarrollo de las competencias básicas en Educación Primaria. Temas para la educación. Revista Digital para profesionales de la enseñanza, 7. Recuperado de https://goo.gl/3fXAqF

McGee, M.G. (1979). Human spatial abilities: psychometric studies and environmental, genetic, hormonal, and neurological influences. Psychological bulletin, 86(5), 889. https://doi.org/10.1037/0033-2909.86.5.889

Melgosa Pedrosa, C., Ramos Barbero, B. \& Baños García, M.E. (2013). Interactive learning management system to develop spatial visualization abilities. Computer Applications in Engineering Education, 21(2), 203-216. https://doi. org/10.1002/cae.21590

Mishra, P. \& Koehler, M. J. (2006). Technological Pedagogical Content Knowledge: A new framework for teacher knowledge. Teachers College Record, 108(6), 1017-1054. https://doi.org/10.1111/j.1467-9620.2006.00684.x

Muro-Medrano, P. (2012). Etapas de la popularización de las infraestructuras de información geoespacial. GeoFocus, Revista Internacional de Ciencia y Tecnología de la Información Geográfica, 12, 1-5. Recuperado de http://geofocus. rediris.es/2012/Editorial_2012.pdf

OCDE. (2010). Working Paper 21st Century Skills and Competences for New Millennium Learners in OECD Countries. EDU Working paper No. 41. Paris: Instituto de Tecnologías Educativas. Recuperado de http://recursostic.educacion.es/ blogs/europa/media/blogs/europa/informes/Habilida des_y_competencias_siglo21_OCDE.pdf

Ontoria, A. (2006). Mapas Conceptuales. Una técnica para aprender. Madrid: Narcea.

Ortega Sánchez, D. y Gómez-Trigueros, I.M. (2017). Las WebQuests y los MOOCs en la enseñanza de las Ciencias Sociales y la formación del profesorado de Educación Primaria. Revista Electrónica Interuniversitaria de Formación del Profesorado, 20(2), 205-220. https://doi.org/10.6018/reifop/20.2.258551

Ortega-Sánchez D. y Gómez-Trigueros I.M. (2019). Didactics of Historical-Cultural Heritage QR Codes and the TPACK Model: An Analytic Revision of Three Classroom Experiences in Spanish Higher Education Contexts. Education Sciences, 9(2), 117. https://doi.org/10.3390/educsci9020117

Pardo, A., Ruiz, M.A. y San-Martín, R. (2015). Análisis de datos en ciencias sociales y de la salud I. Madrid: Síntesis.

Raykov, T. \& Marcoulides, G.A. (2017). Ecuation of true criterion validity for unidimensional multicomponent measuring instruments in longitudinal studies. Structural Equation Modeling, 24(4), 599-608. https://doi.org/10.1080/1070551 1.2016 .1172486

Real Decreto 126/2014, de 28 de febrero, por el que se establece el currículo básico de la Educación Primaria y se fijan sus enseñanzas mínimas. BOE n 52, martes 1 marzo 2014. pág. 19349. Recuperado de http://www.boe.es/boe/ dias/2014/03/01/pdfs/BOE-A-2014-2222.pdf

Real Decreto 1105/2014, de 26 de diciembre, por el que se establece el currículo de la E.S.O. y Bachillerato, BOE n 3 , 3 de enero de 2015, pág. 169. Recuperado de https://www.boe.es/boe/dias/2015/01/03/pdfs/BOE-A-2015-37.pdf

Rychen, D.S., Hersh, L. y Userkonstant, J. (2003). Definición y Selección de las Competencias (DeSeCo): Fundamentos teóricos y conceptuales de las competencias. París: OCDE.

Rychen, D. S. \& Tiana, A. (2004). Developing key competencies in education: some lessons from international and national experience. Ginebra: UNESCO-IBE, Studies in Comparative Education. 
Richards, J.C. \& Rogers, S.T. (2001). Approaches and Methods in Language Teaching. Cambridge: Cambridge University Press. https://doi.org/10.1017/CBO9780511667305

Roig-Vila, R., Mengual-Andrés, S., Sterrantino, C. y Quinto, P. (2015). Actitudes hacia los recursos tecnológicos en el aula de los futuros docentes. @tic. Revista d'innovació educativa, 15, 12-19. https://doi.org/10.7203/attic.15.7220

Sánchez-Gómez, M. C., Rodrigues, A. I. y Costa, A. P. (2018). Desde los métodos cualitativos hacia los modelos mixtos: tendencia actual de investigación en ciencias sociales. Revista Ibérica de Sistemas e Tecnologias de Informação, 28, 9-13. https://doi.org/10.17013/risti.28.0

Schelly, C., Anzolone, G., Wijnen, B. \& Pearce, J.M. (2015). Open-source 3-D printing technologies for education: bringing additive manufacturing to the classroom. J. Visual Lang. Comput. 28, 226-237. https://doi.org/10.1016/j. jvlc.2015.01.004

Schmidt, D. A., Baran, E., Thompson A. D., Koehler, M. J., Mishra, P., \& Shin, T. (2009). Technological pedagogical content knowledge (tpack): The development and validation of an assessment instrument for preservice teachers. Journal of Research on Technology in Education, 42(2), 123-149. https://doi.org/10.1080/15391523.2009.10782544

Shulman, S. L. (1987). Knowledge and Teaching. Foundations of the New Reform. Harvard Educational Review, $57,1-22$. https://doi.org/10.17763/haer.57.1.j463w79r56455411

Torner, J., Alpiste, F. \& Brigos, M. (2014). Spatial Ability in Computer-Aided Design Courses. Computer-Aided Design and Applications, 12(1) 36-44. http://dx.doi.org/10.1080/16864360.2014.949572

Tsou, M.H. \& Yanow, K. (2010). Enhancing General Education with Geographic Information Science and Spatial Literacy. URISA Journal 22(2), 45-55. Recuperado de https://geoinfo.sdsu.edu/hightech/Images/URISAjournal/2010-Tsou-Yanow-URISA\%20Journal\%20Vol.22\%20Issue\%202.pdf

UNESCO (1996). La educación encierra un tesoro, informe a la UNESCO de la Comisión Internacional sobre la Educación para el Siglo XXI. París, UNESCO. https://doi.org/10.22201/fcpys.2448492xe.1996.166.49503

UNESCO. (1998). Declaración Mundial Sobre la Educación Superior en el Siglo XXI. Visión y Acción. París: Editorial UNESCO.

UNESCO. (2007). Enfoque por Competencias, Oficina Internacional de Educación. Recuperado de http://www.ibe.unesco. org/es/comunidades/comunidad-de-practicacop/enfoque-por-competencias.html.

UNESCO. (2013). Las competencias en el desarrollo curricular. Recuperado de http://www.ibe.unesco.org/cops/Competencies/Competencias_esp.pdf.

UNESCO (2016). Educación para la Ciudadanía Mundial. Preparar a los educandos para los retos del siglo XXI. París. Recuperado de http://unesdoc.unesco.org/images/0024/002449/244957s.pdf

Vázquez Maris, S. y Noriega Biggio, M. (2010). La competencia espacial. Evaluación en alumnos de nuevo ingreso a la universidad. Educación Matemática, 22(2), 65-91.

Vázquez Maris, S., Noriega Biggio, M. y Maris García, S. (2013). Relaciones entre rendimiento académico, competencia espacial, estilos de aprendizaje y deserción. REDIE, 15(1), 29-44.

Villa Sicilia, A. (2016). Desarrollo y evaluación de las habilidades espaciales de los estudiantes de Ingeniería. Actividades y estrategias de resolución de tareas espaciales. Tesis Doctoral. Universitat Politécnica de Catalunya.

Wai, J., Lubinski, D. \& Benbow, C.P., (2009). Spatial ability for STEM domains: Aligning over 50 years of cumulative psychological knowledge solidifies its importance. Journal of educational psychology, 101(4), 817-821. https://doi. org/10.1037/a0016127 